\title{
Characterization of the temperature of free electrons diffusing from a magnetically expanding current-free double layer plasma
}

\author{
Kazunori Takahashi ${ }^{1-3)}$, Christine Charles ${ }^{1)}$, Rod Boswell ${ }^{1)}$, Michael A. Lieberman ${ }^{1,4)}$, and Rikizo Hatakeyama ${ }^{2)}$

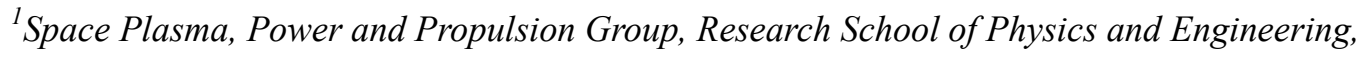 \\ The Australian National University, Canberra 0200, Australia \\ ${ }^{2}$ Department of Electronic Engineering, Tohoku University, Sendai 980-8579, Japan \\ ${ }^{3}$ Department of Electrical and Electronic Engineering, Iwate University, Morioka 020-8551, Japan \\ ${ }^{4}$ Department of Electrical Engineering and Computer Sciences, University of California, Berkeley CA 94720 , \\ USA. \\ Email: kazunori@iwate-u.ac.jp
}

\begin{abstract}
Electron temperature is characterized in a low-pressure argon plasma diffusing from a magnetically expanding radiofrequency plasma containing a current-free double layer (DL) over the argon gas pressure of $0.3-3$ mTorr. The measured electron energy probability function (EEPF) is Maxwellian at the downstream side of the DL, while the upstream EEPF shows a depleted tail corresponding to free electrons, which can overcome the potential drop of the DL and diffuse into the downstream side. The free electrons relating to the amplitude of the DL electrically neutralize a supersonic ion beam accelerated by the DL. The electron temperature measured at the downstream plasma is found to be in good agreement with that of the free electrons in the upstream source tube and agrees with the numerical result given from low-pressure diffusion theory.
\end{abstract}


Formation of electric double layers (DLs) in plasmas has recently been a vigorous subject of research associated with particle acceleration in space plasmas [1,2], with confinement of fusion plasmas [3], and with development of plasma propulsion devices $[4,5]$. In earlier laboratory experiments, the energy distributions of ions and electrons were measured in a multiple plasma machine operated in a double plasma mode [6, 7], where there are two plasma sources at the axial ends of the machine. The results showed both trapped particles (reflected by the DL) and free particles (accelerated by and overcoming the DL).

In recent experiments on the magnetically expanding low-pressure DL plasma, the spontaneous formation of a DL is observed in a single-source system [8]. The ion energy distribution functions (IEDFs) obtained in experiments and in 1D particle-in-cell (PIC) simulations have demonstrated a supersonic ion beam accelerated by the potential drop of the DL $[9,10,11,12,13,14,15]$. The electron energy distributions have also been observed in the PIC simulations and in experiments using an rf-compensated Langmuir probe $[16,17,18,19]$. The observed electron energy distribution or probability functions (EEDFs or EEPFs) upstream of the DL have a depleted tail above a break energy corresponding to the potential drop of the DL [17]. Upstream source electrons with energy lower and higher than the break energy can be reflected and overcome the potential drop of the DL, respectively, i.e., they act as trapped and free electrons. On the other hand, it has been observed that the EEPF downstream of the DL is Maxwellian with the temperature of the free electrons [17]. The amplitudes of the DL potential drop and the ion acceleration can commonly be evaluated by the temperature of the free electrons. In magnetically expanding double layer plasmas, hence, the temperature of the downstream electrons overcoming the DL and diffusing from the upstream source is a key parameter for understanding the behavior of the DL.

In the present communication, the electron temperature downstream of the DL is experimentally characterized by combination of an rf-compensated probe and a pulsed probe technique in a magnetically expanding double-layer plasma. The results are compared with numerical results given from low-pressure diffusion theory $[20,21,22]$.

The Chi-Kung experimental setup has been described previously [10] and is shown in Fig. 1. It consists of a 31-cm-long, 13.7-cm-diameter helicon source attached contiguously to a grounded 29.4-cm-long, 31.8-cm-diameter diffusion chamber at a base pressure of a few $10^{-6}$ Torr. The argon gas is introduced via a 
chamber side port and held at an operating pressures $P_{\mathrm{Ar}}$ in the range of 0.3-3 mTorr, measured using a Baratron gauge. The source consists of a Pyrex glass tube surrounded by a double-saddle antenna supplied with $250 \mathrm{~W}$ radiofrequency power at $13.56 \mathrm{MHz}$. Surrounding the source tube and antenna, two solenoids placed at $z=3 \mathrm{~cm}$ and $21 \mathrm{~cm}$ produce a magnetic field diverging from a maximum of about $130 \mathrm{G}$ in the source to a few tens of gauss in the diffusion chamber. The calculated diverging magnetic-field lines are shown in Fig. 1 as solid lines. In the present machine, the DL spontaneously forms near the open end of the source tube at $z=25 \mathrm{~cm}$ [8] with a potential drop of about $25 \mathrm{~V}$ for $P_{\mathrm{Ar}}=0.3$ mTorr [23]. Simultaneously with the formation of the DL, a supersonic ion beam with energy corresponding to the potential drop is detected in the diffusion chamber.

The measurement of the EEPF in rf plasmas is very difficult because the I-V curve of the probe is distorted by the rf oscillation of the plasma potential. In order to measure the EEPF, an axially movable, $3 \mathrm{~mm}$-long 0.25 mm-diam cylindrical, rf-compensated Langmuir probe (CP) shown in Fig. 2(a) is inserted from the vacuum port connected to the downstream flange. The design of the rf-compensated probe is described in Ref. [24]. The probe tip is connected to five choke coils with impedance resonances at the fundamental $(13.56 \mathrm{MHz})$ and second-harmonics $(27.12 \mathrm{MHz})$ frequencies. A reference electrode is also connected to the probe tip through a 4.7 $\mathrm{nF}$ capacitor. Then, the $\mathrm{rf}$ potential oscillation detected by the reference electrode is superimposed on the probe bias voltage; thereby the I-V curve distorted by the rf oscillation is compensated. The circuit diagram of the EEPF measurement is shown in Fig. 2(b). The probe bias voltage is swept at about $10 \mathrm{~Hz}$ from -120 to $+120 \mathrm{~V}$ and the signal of the probe current is detected through a resistor connected to a bipolar power supply. The signal is fed through an isolation amplifier and twice differentiated by the active circuit described as an inset in Fig. 2(b). The circuit acts both as a differentiator for the low frequency signal below several hundreds $\mathrm{Hz}$ and as a rejecting filter for plasma instabilities above a few $\mathrm{kHz}$. The above-mentioned pulsed probe technique has been reported in previous work [25]. The differentiated signal is digitized at $50 \mathrm{kHz}$ and 14 bits, yielding the EEPF.

Figure 2(c) shows the EEPFs upstream and downstream of the DL, measured at $z=17 \mathrm{~cm}$ (red line) and $z=36$ cm (blue line) for $P_{\mathrm{Ar}}=0.3$ mTorr, which has already been reported by the authors in Ref. [17]. The upstream EEPF in Fig. 2(c) clearly shows a break energy $\varepsilon_{\text {break }}$ in the slope at about $27 \mathrm{eV}$ as indicated by the arrow. The observed $\varepsilon_{\text {break }}$ closely corresponds to the previously measured potential drop of the DL and the energy of the 
subsequent ion beam [23]. In the EEPF, the straight line in the logarithmic scale indicates a Maxwellian distribution and its slope gives the electron temperature. Below $\varepsilon_{\text {break }}$, the slope of the EEPF yields a temperature of about $8 \mathrm{eV}\left(T_{\mathrm{e} \text { trapped }}\right)$, whereas the temperature of the electrons above $\varepsilon_{\text {break }}$ is about $5 \mathrm{eV}\left(T_{\mathrm{e} \text { free }}\right)$. The electrons with energy lower than $\varepsilon_{\text {break }}$ having a high-temperature population $\left(T_{\mathrm{e}}\right.$ trapped $)$ are electrostatically trapped by the potential drop of the DL and the sheath voltages at the left-side floating wall. Meanwhile the high-energy electrons above $\varepsilon_{\text {break }}$ with lower temperatures $\left(T_{\mathrm{e}}\right.$ free $)$ can overcome the potential drop of the DL and diffuse into the downstream plasma; hence they act as free electrons and neutralize the supersonic ion beam accelerated by the DL. In fact, the EEPF downstream of the DL, shown as a blue line in Fig. 2(c), is Maxwellian and resembles that of the upstream depleted-tail population. In the downstream plasma diffusing from the magnetically expanding, DL-containing plasma, thus, only free electrons are detectable. The slope of the downstream EEPF gives a temperature $\left(T_{\mathrm{e} \text { down }}\right)$ of about $5 \mathrm{eV}$, which is very close to $T_{\mathrm{e}}$ free.

Detailed measurements of the three electron temperatures $\left(T_{\mathrm{e} \text { trapped }}, T_{\mathrm{e} \text { free, }}, T_{\mathrm{e} \text { down }}\right)$ have been performed for various operating gas pressures over the range of $0.3-3$ mTorr. The measured $T_{\mathrm{e}}$ trapped, $T_{\mathrm{e}}$ free, and $T_{\mathrm{e} \text { down }}$ are plotted in Fig. 3 as closed circles, open circles, and closed squares, respectively. Over the gas pressure range in the present experiments, the downstream electron temperature $T_{\mathrm{e} \text { down }}$ corresponds to that of the free electrons $\left(T_{\mathrm{e}}\right.$ free $)$ observed in the upstream source. Hence, we can deduce that only the upstream, energetic, depleted tail electrons can diffuse into the downstream side by overcoming the DL potential drop, and that the temperature of the downstream electrons shows that of the free electrons passing through the DL.

With an increase in $P_{\mathrm{Ar}}$ in Fig. 3, the temperature ( $\left.T_{\mathrm{e} \text { free, }}, T_{\mathrm{e} \text { down }}\right)$ of the free electrons decreases from about 5 $\mathrm{eV}$ to about $2.5 \mathrm{eV}$ due to the changing ionization balance. The measured temperatures are compared with the numerical result given from low-pressure diffusion theory [20], which has also been used to estimate the temperature of the Maxwellian electrons in an analytical model of the current-free DL $[21,22]$. In the model, the downstream particle balance is described by equating the total surface particle loss to the total ionization as [20, 22]

$$
K_{i z} n_{g} \pi R_{1}^{2} \cdot 2 w=\left(2 \pi R_{1}^{2} h_{2 w}+2 \pi R_{1} \cdot 2 w h_{R 1}\right) u_{B}
$$

where $K_{\mathrm{iz}}\left(T_{\mathrm{e}}\right)$ is the ionization rate coefficient for Maxwellian electrons, $n_{\mathrm{g}}$ is the neutral gas density, 


$$
h_{2 w}=0.86\left(3+w / \lambda_{i}\right)^{-1 / 2}, \quad h_{R 1}=0.8\left(4+R_{1} / \lambda_{i}\right)^{-1 / 2},
$$

are the axial and radial center-to-edge density ratios derived from the low-pressure diffusion theory [20], and $\lambda_{\mathrm{i}} \sim$ $\left(330 P_{\mathrm{Ar}}\right)^{-1}$ is the ion mean free path in argon, with $\lambda_{\mathrm{i}}$ in centimeters and $P_{\mathrm{Ar}}$ in Torr. The geometrical configuration of the experimental machine can be characterized by the length $w$ and radius $R_{1}$ of the diffusion chamber. Because the experimental data indicates a density maximum near the source/diffusion chamber junction, with small axial losses to this surface, the effective length of the diffusion chamber in Eqs. (1) and (2) has been chosen to be $2 w$. By solving Eq.(1) numerically, the temperature of the Maxwellian electrons in the diffusion chamber can be obtained for various gas pressures $P_{\mathrm{Ar}}$. The numerical result, which has already been shown in Fig. 4(b) of Ref. [22], is plotted in Fig. 3 as a solid line with open triangles, where the result is essentially unrelated to the formation of a DL, and is a global balance in the low-pressure diffusion chamber. The numerical result $T_{\mathrm{e} \text { model }}$ is found to agree well with the experimentally obtained temperature of the free electrons $\left(T_{\mathrm{e}}\right.$ free, $\left.T_{\mathrm{e} \text { down }}\right)$. Therefore, the present results show that global low-pressure diffusion theory yields the temperature of the free electrons in the magnetically expanding DL plasma.

In summary, the temperatures of the upstream-free and downstream electrons are experimentally characterized in the magnetically expanding, rf plasmas containing a current-free DL over the range of the gas pressure of $0.3-$ 3 mTorr, where the electron energy probability functions upstream and downstream of the DL are measured by a combination of an rf-compensated probe and a pulsed probe technique. The downstream electrons correspond to the upstream, energetic free electrons overcoming the DL and diffusing into the diffusion chamber; the energy distributions are actually observed to match well $\left(T_{\mathrm{e} \text { down }}=T_{\mathrm{e} \text { free }}\right)$. It is found that the temperatures can be well described by a global model using low-pressure diffusion theory. 


\section{List of references}

[1] Ergun R E, Su Y -J, Andersson L, Carlson C W, McFadden J P, Mozer F S, Newman D L, Goldman M V and Strangeway R J 2001 Phys. Rev. Lett. 87, 045003

[2] Boswell R W, Marsch E and Charles C 2006 Astrophys. J. 640, L199

[3] Takahashi K, Kaneko T and Hatakeyama R 2007 Appl. Phys. Lett. 91, 261502

[4] Charles C 2007 Plasma Sources Sci. Technol. 16, R1

[5] Charles C 2009 J. Phys. D: Appl. Phys. 42, 163001

[6] Quon B H and Wong A Y 1976 Phys. Rev. Lett. 37, 1393

[7] Leung P, Wong A Y and Quon B H 1979 Phys. Fluids 23, 992

[8] Charles C and Boswell R W 2003 Appl. Phys. Lett. 82, 1356

[9] Cohen S A, Siefert N S, Stange S, Boivin R F, Scime E E, Levinton F M 2003 Phys. Plasmas 10, 2593

[10] Charles C and Boswell R W 2004 Phys. Plasmas 11, 1706

[11] Sun X, Keesee A M, Biloiu C, Scime E E, Meige A, Charles C and Boswell R W 2005 Phys. Rev. Lett. 95, 025004

[12] Meige A, Boswell R W, Charles C and Turner M M 2005 Phys. Plasmas 12, 052317

[13] Biloiu I A, Scime E E and Biloiu C 2008 Appl. Phys. Lett. 92, 191502

[14] Takahashi K, Oguni K, Yamada H and Fujiwara T 2008 Phys. Plasmas 15, 084501

[15] Takahashi K and Fujiwara T 2009 Appl. Phys. Lett. 94, 061502

[16] Meige A and Boswell R W 2006 Phys. Plasmas 13, 092104

[17] Takahashi K, Charles C, Boswell R W, Kaneko T and Hatakeyama R 2007 Phys. Plasmas 14, 114503

[18] Takahashi K, Charles C, Boswell R W and Hatakeyama R 2008 Phys. Plasmas 15, 074505

[19] Takahashi K, Charles C, Boswell R W, Cox W and Hatakeyama R 2009 Appl. Phys. Lett. 94, 191503

[20] Lieberman M A and Lichtenberg A J 2005 Principle of Plasma Discharges and Materials Processing 2nd ed.

(Wiley-Interscience) Chap. 10.2

[21] Lieberman M A and Charles C 2006 Phys. Rev. Lett. 97, 045003

[22] Lieberman M A, Charles C and Boswell R W 2006 J. Phys. D: Appl. Phys. 39, 3294

[23] Charles C and Boswell R W 2007 Appl. Phys. Lett. 91, 201505

[24] Sudit I D and Chen F F 1994 Plasma Sources Sci. Technol. 3, 162

[25] Schoenberg K F 1978 Rev. Sci. Instrum. 49, 1377 


\section{List of figure captions}

Figure 1:

Schematic of the Chi-Kung machine operated for the magnetically expanding double layer plasma mode.

Figure 2:

(a) Design of the rf-compensated probe. (b) Circuit diagram of the pulsed probe technique.

(c) Representative electron energy probability functions upstream $(z=17 \mathrm{~cm}$, red line) and downstream $(z=36$ $\mathrm{cm}$, blue line) of the DL. The solid arrow shows the break energy $\varepsilon_{\text {break }}$. The dashed lines show the tangential lines giving the electron temperatures ( $\left.T_{\mathrm{e} \text { trapped }}, T_{\mathrm{e} \text { free }}, T_{\mathrm{e} \text { down }}\right)$.

(This figure is in color only in the electronic version)

Figure 3:

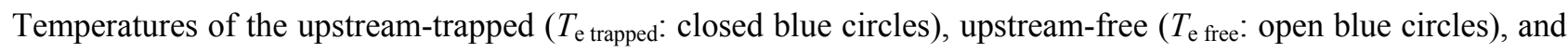
downstream ( $T_{\mathrm{e} \text { down }}$ : closed red squares) electrons, together with the numerical electron temperature $\left(T_{\mathrm{e} \text { model }}\right.$ : solid line with open triangles) calculated by Eq. (1) [22].

(This figure is in color only in the electronic version) 


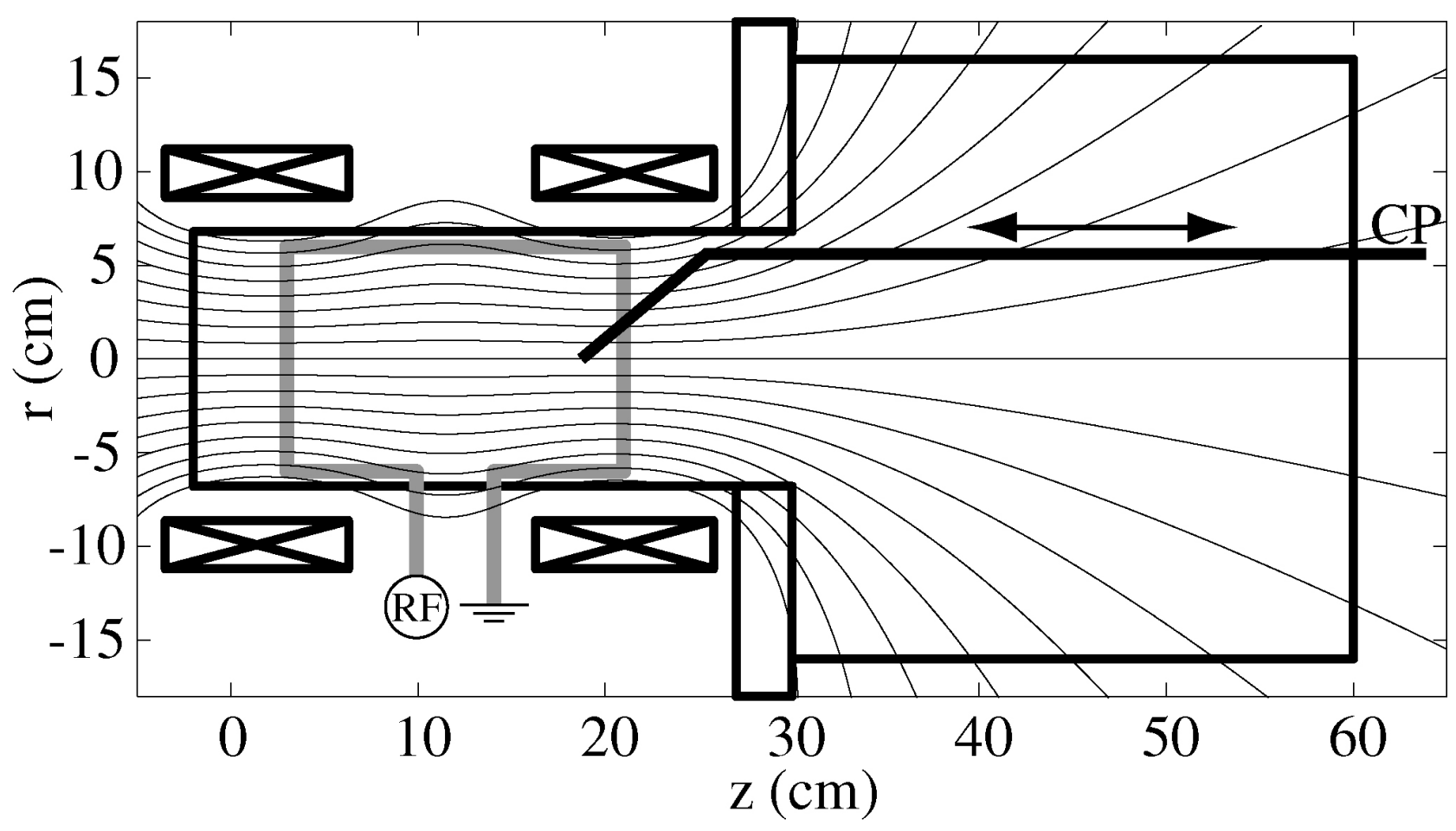

Figure 1 
(a)

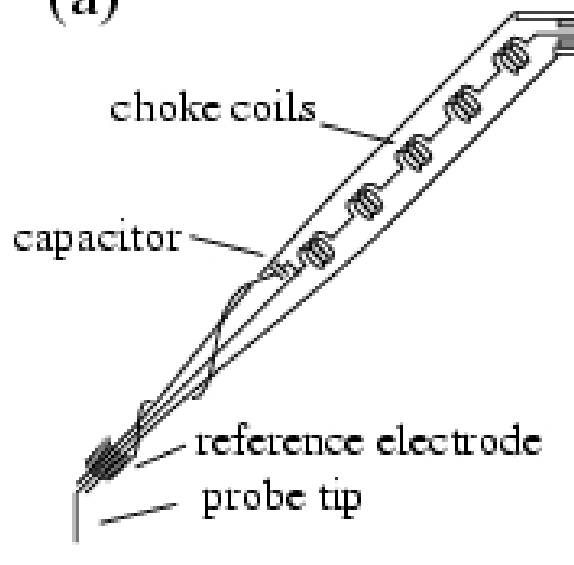

(b)

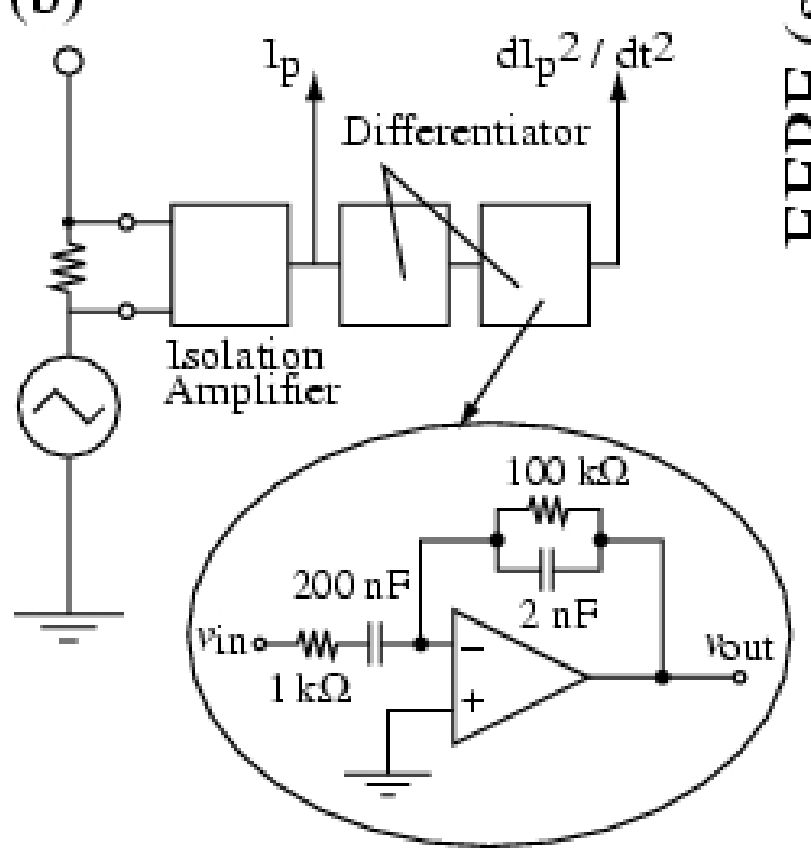

(c)

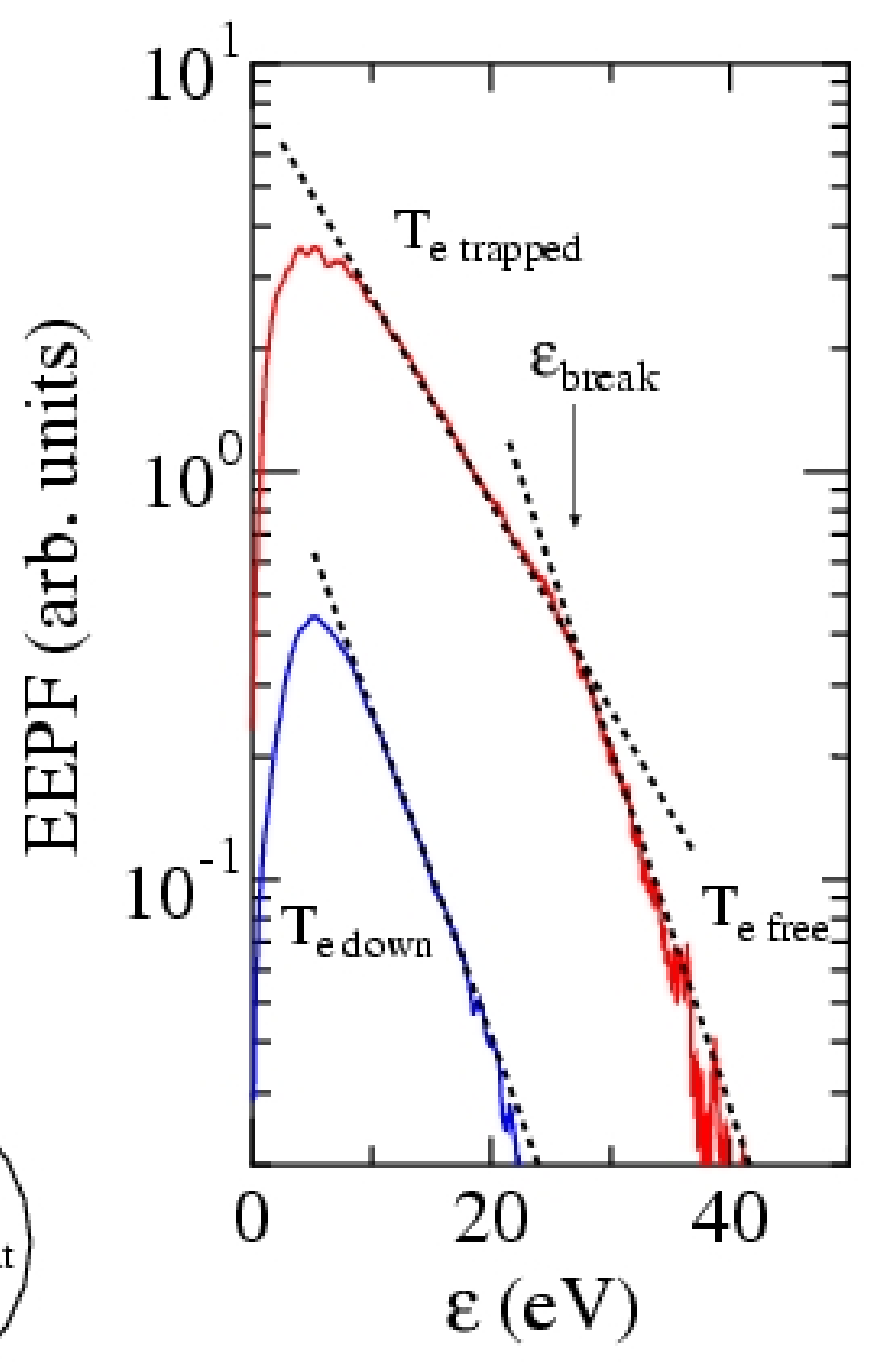

Figure 2 


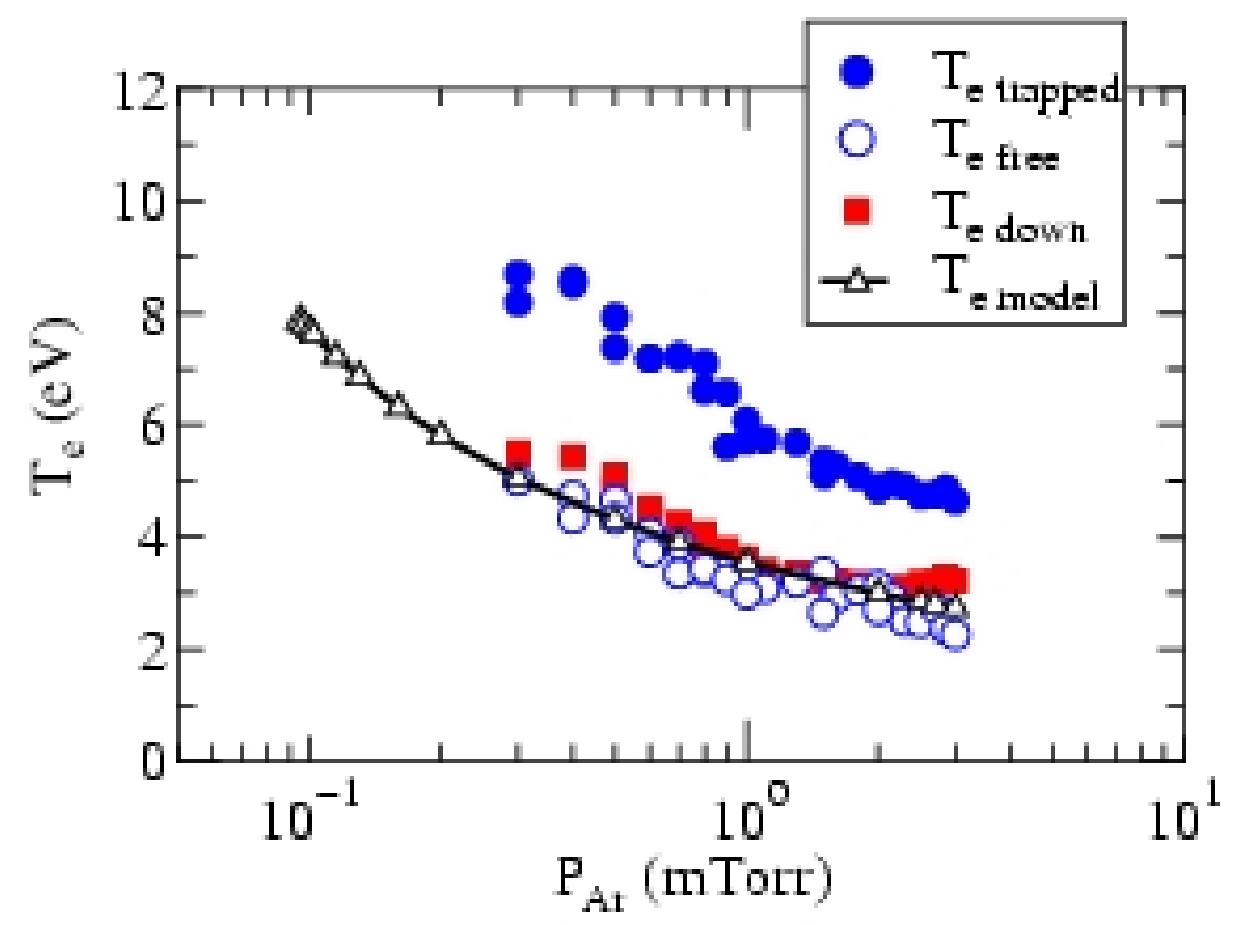

Figure 3 